\title{
Combination of Serine Protease Inhibitor FUT-175 and Thromboxane Synthetase Inhibitor OKY-046 Decreases Cerebral Vasospasm in Patients with Subarachnoid Hemorrhage
}

\author{
Makio KAMINOGO, Masahiro YONEKURA*, Masanari OnIZUKA, \\ Akio YASUNAGA, and Shobu SHIBATA
}

\begin{abstract}
Department of Neurosurgery, Nagasaki University School of Medicine, Nagasaki; ${ }^{*}$ Department of Neurosurgery, Nagasaki National Central Hospital, Nagasaki
\end{abstract}

\begin{abstract}
The preventive effect of the serine protease inhibitor FUT-175 (nafamostat mesilate), a potent inhibitor of the complement system, against vasospasm was evaluated in 34 high risk patients with thick and diffuse subarachnoid hemorrhage (SAH) demonstrated by computed tomography corresponding to Fisher group 3. All patients underwent surgery within 96 hours following SAH and received the thromboxane $A_{2}$ synthetase inhibitor, OKY-046, as part of standard care. FUT-175 (40-160 mg/day) was administered during the initial 4 days following surgery. 455 patients treated without FUT-175 in the Nagasaki SAH Data Bank (non-FUT group) formed the control group. FUT-175 significantly decreased the incidence of symptomatic vasospasm in patients with severe neurological grade (Hunt and Hess grade 3, $p<0.02 ;$ Hunt and Hess grade $4, p<0.02$ ). The incidence of favorable outcome was $76.5 \%$ in the FUT group and $60.4 \%$ in the non-FUT group, but not statistically different. However, when patients of Hunt and Hess grade 5 were excluded, the FUT group had a significantly improved outcome $(p<0.05)$. This study suggests that FUT-175 has an additive effect to OKY-046 in preventing vasospasm in high risk patients with severe $\mathrm{SAH}$.
\end{abstract}

Key words: cerebral aneurysm, serine protease inhibitor, subarachnoid hemorrhage, thromboxane $\mathrm{A}_{2}$ synthetase inhibitor, vasospasm

\section{Introduction}

Delayed ischemic neurological deficit (DIND) due to chronic cerebral vasospasm continues to be the major cause of poor clinical outcome in patients with subarachnoid hemorrhage (SAH). Many therapeutic agents are in use to prevent or reduce vasospasm, , 1,2,6,14,20,21) but no definitive treatment has been identified. Recent experimental and clinical studies suggest that the inflammatory process is important in the pathogenesis of vasospasm. Histological examination has demonstrated infiltration of neutrophils, lymphocytes, plasma cells, and macrophages into spastic arterial walls in humans ${ }^{103}$ and experimental animals ${ }^{22)}$ as well as deposition of immunoglobulin $\mathrm{G}$ and the complement component C3.9) Furthermore, within 48 hours of SAH, sig-

Received February 16, 1998; Accepted June 26, 1998 nificant activation of the complement components C3 and C4 occurred in the cerebrospinal fluid [CSF] space of patients who later developed DIND. ${ }^{15]}$ The elevated levels of these complement components in the early post-SAH period fell rapidly within a few days. ${ }^{15)}$ Also, a high serum concentration of immunocomplexes precedes the onset of vasospasm. ${ }^{18)}$ These observations support the hypothesis that the inflammatory process, particularly activation of the complement system, may trigger vasospasm, and therefore treatment with FUT-175 (nafamostat mesilate), an inhibitor of the complement system, ${ }^{8,12]}$ in the very early post-SAH period will prevent vasospasm. ${ }^{23,24)}$ The amount of clot in the basal cistern is a risk factor for chronic vasospasm ${ }^{3.4)}$ and the incidence of symptomatic vasospasm is greater than $50 \%$ in patients with thick SAH on computed tomography [CT]. ${ }^{4,5]}$

This study evaluated the use of FUT-175 in addi- 
tion to a thromboxane $\mathrm{A}_{2}\left(\mathrm{TXA}_{2}\right)$ synthetase inhibitor OKY-046 (sodium ozagrel) for the treatment of high risk patients with thick and diffuse SAH on CT.

\section{Materials and Methods}

This study included 34 patients with diffuse and thick clot in the subarachnoid space (Fisher group $3^{33}$ ) who underwent clipping of the aneurysm within 96 hours of first onset of symptoms between 1990 and 1995 (FUT group). Patients with intracerebral or intraventricular clot as well as diffuse and thick clot in the subarachnoid space were also included. Informed consent was obtained from the patients or guardians. Continuous intravenous infusion of OKY-046 (80 mg/day) was carried out from the day following surgery to day 14 of SAH. FUT-175 (40$160 \mathrm{mg} /$ day, divided into $2-4$ doses) was administered intravenously for the initial 4 days following surgery. The rate of infusion was approximately $20 \mathrm{mg} / 60 \mathrm{~min}$.

The aim of the present study was to evaluate the effect of additional use of FUT-175 to OKY-046 for the prevention of vasospasm. Therefore, 455 patients classified in Fisher group 3, and who underwent surgery within 96 hours following $\mathrm{SAH}$, followed by treatment with OKY-046 from the Nagasaki SAH Data Bank formed the control group (non-FUT group). Patients who had new neurological deficits immediately after the operation were excluded. Other treatments for vasospasm such as hypertensive hypervolemic therapy, low molecular weight dextran, mannitol, and glycerol were allowed. Calcium antagonist to control blood pressure was also allowed. However, aggressive removal of subarachnoid clots during surgery was not usually employed in either the FUT group or the non-FUT group.

Comparison of the characteristics of the patients, including age, sex, neurological severity of SAH, location of aneurysm, and timing of surgery between the two groups found no statistical difference (Table 1). Cisternal drainage was placed in all patients in the FUT group. CSF drainage was not used in 40 patients and only ventricular drainage in 21 patients in the non-FUT group. The cisternal drainage and/or the spinal drainage was placed in the remaining 394 cases in the non-FUT group.

The diagnosis of symptomatic vasospasm was based on exclusion of other causes of delayed neurological deficit such as rebleeding, hydrocephalus, electrolyte disturbance, and seizure. ${ }^{1,7,19]}$ Overall outcome was assessed 3 months following SAH using the Glasgow Outcome Scale. ${ }^{19)}$ Transient vasospasm indicated complete recovery of DIND within 3
Table 1 Characteristics of patients

\begin{tabular}{|c|c|c|}
\hline Factor & FUT group & Non-FUT group \\
\hline No. of patients & 34 & 455 \\
\hline male & $10(29.4)$ & $162(35.6)$ \\
\hline female & $24(70.6)$ & $293[64.4]$ \\
\hline Mean age (yrs) & $60.6 \pm 13.4$ & $60.5 \pm 11.7$ \\
\hline \multicolumn{3}{|c|}{ Hunt and Hess grade } \\
\hline I & $1(2.9)$ & $18(4.0)$ \\
\hline II & $6(17.6)$ & $134(29.5)$ \\
\hline III & $16(47.1)$ & $204(44.8)$ \\
\hline IV & $9(26.5)$ & 86 (18.9) \\
\hline $\mathrm{V}$ & $2[5.9]$ & $13(2.9)$ \\
\hline \multicolumn{3}{|c|}{ Location of aneurysm } \\
\hline ACoA & $9(26.5)$ & $161(35.4)$ \\
\hline ICA & $10(29.4)$ & $119(26.2)$ \\
\hline MCA & $14(41.2]$ & $134(29.5)$ \\
\hline $\mathrm{ACA}$ & $1(2.9)$ & $28(6.2)$ \\
\hline $\mathrm{BA}$ & 0 & $6(1.3)$ \\
\hline others & 0 & $7(1.5)$ \\
\hline \multicolumn{3}{|c|}{ Aneurysm clipping } \\
\hline day 0 & $11(32.4)$ & $150(33.0)$ \\
\hline day 1 & $20(58.8)$ & $209(45.9)$ \\
\hline day 2 & $2(5.9)$ & $63(13.8)$ \\
\hline day 3 & $1(2.9)$ & $33(7.3)$ \\
\hline
\end{tabular}

Values in parentheses indicate percentages. Statistical values are expressed as mean \pm SD. ACA: anterior cerebral artery, ACoA: anterior communicating artery, BA: basilar artery, day 0: day of subarachnoid hemorrhage, ICA: internal carotid artery, MCA: middle cerebral artery.

\section{months.}

Statistical values are expressed as mean $\pm S D$, and were compared with the unpaired t-test. Comparison of background characteristics, incidence of vasospasm, and clinical outcome between the two groups used the chi-square test. A significant statistical difference was defined as $\mathrm{p}<0.05$.

\section{Results}

Administration of FUT-175 significantly decreased the overall incidence of symptomatic vasospasm including permanent and transient vasospasm ( $\mathrm{p}<$ 0.001 , Table 2). Administration of FUT-175 significantly decreased the incidence of symptomatic vasospasm in patients with Hunt and Hess ${ }^{11)}$ grade 3 ( $p<0.02$ ) and grade 4 ( $p<0.02$ ). The incidence of symptomatic vasospasm in patients with grade 1 plus 2 was also decreased but no statistical difference was found.

Patients who had received FUT-175 tended to achieve a more favorable outcome (good recovery or moderate disability), but this difference was not 
Table 2 Incidence of symptomatic vasospasm in the FUT group and non-FUT group

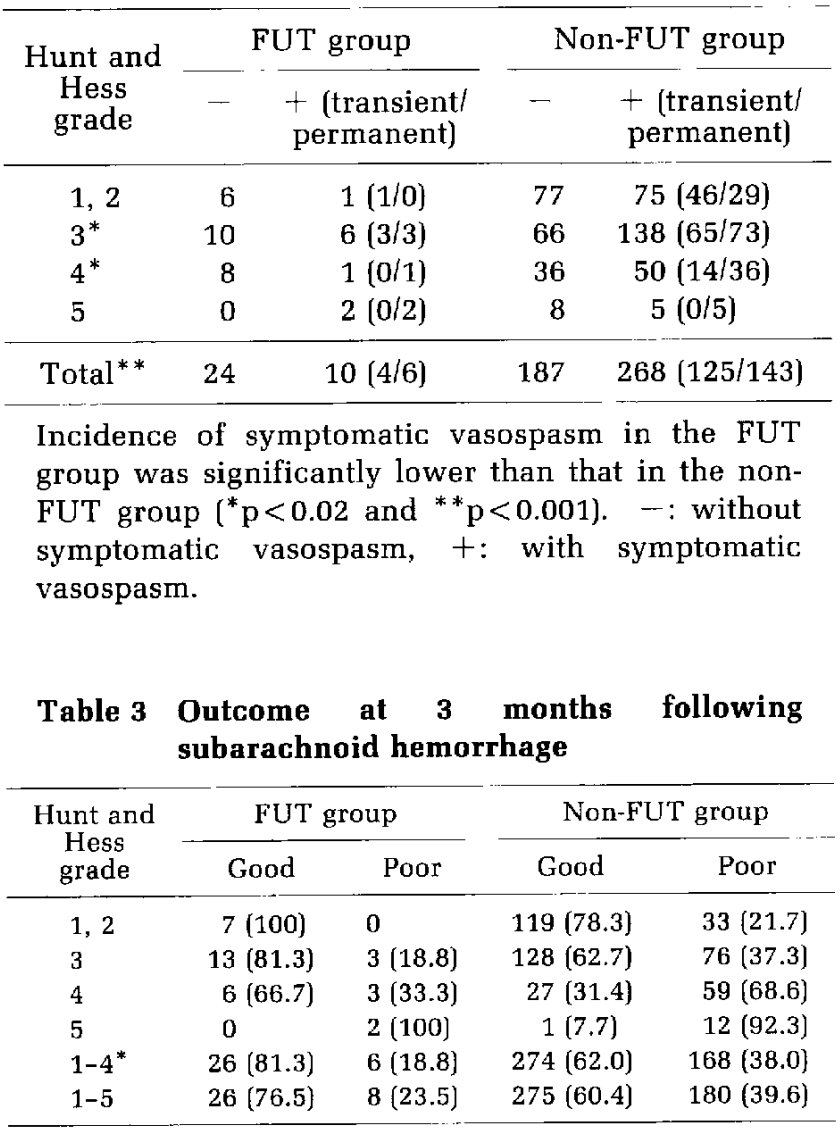

Values in parentheses indicate percentages. Incidence of good outcome was significantly higher in the FUT group ( $\left.{ }^{*} \mathrm{p}<0.05\right)$. Good: good recovery or moderate disability; poor: severe disability, vegetative state, or dead.

statistically significant (Table 3). However, if patients with grade 5 who suffered from serious neurological deficit caused by the direct effect of SAH were excluded, 26 of 32 patients in the FUT group (81.3\%) showed favorable outcome, which was significantly higher than the $62.0 \%$ in the non-FUT group $(p<0.05)$.

Two patients were given less than $80 \mathrm{mg} /$ day of FUT-175 in the initial study, but both developed symptomatic vasospasm (Fig. 1). FUT-175 was initiated on day 2 of $\mathrm{SAH}$ in one patient and on day 3 of SAH in another. In the subsequent study, a dose of $80 \mathrm{mg} /$ day or higher was used. The incidence of symptomatic vasospasm decreased from $29.4 \%$ to $25 \%$ in the FUT group after exclusion of these two patients. Only three of 13 patients treated with 80 mg/day FUT-175 exhibited symptomatic vasospasm, whereas five of 19 patients who received more than $80 \mathrm{mg} /$ day of FUT-175 developed symptomatic

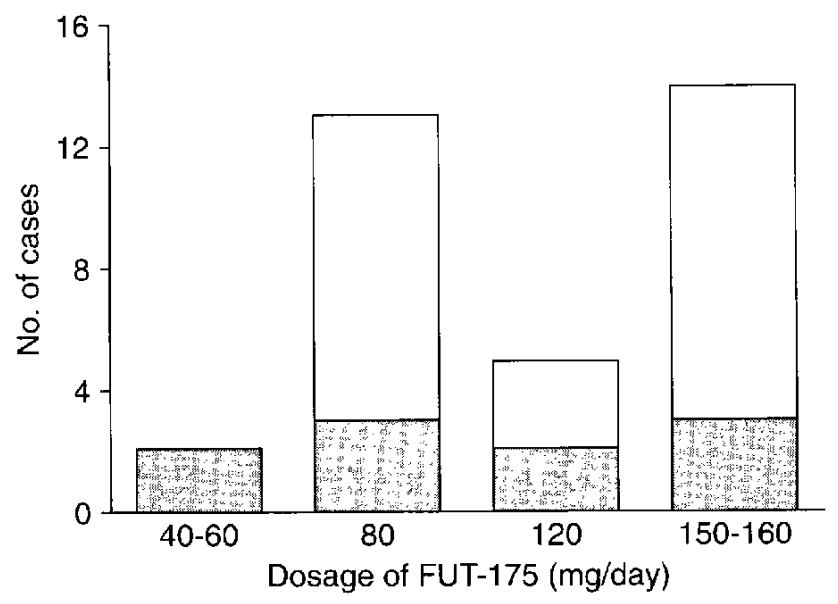

Fig. 1 Dosage of FUT-175 and incidence of symptomatic vasospasm. open column: patients without symptomatic vasospasm, shaded column: patients with symptomatic vasospasm.

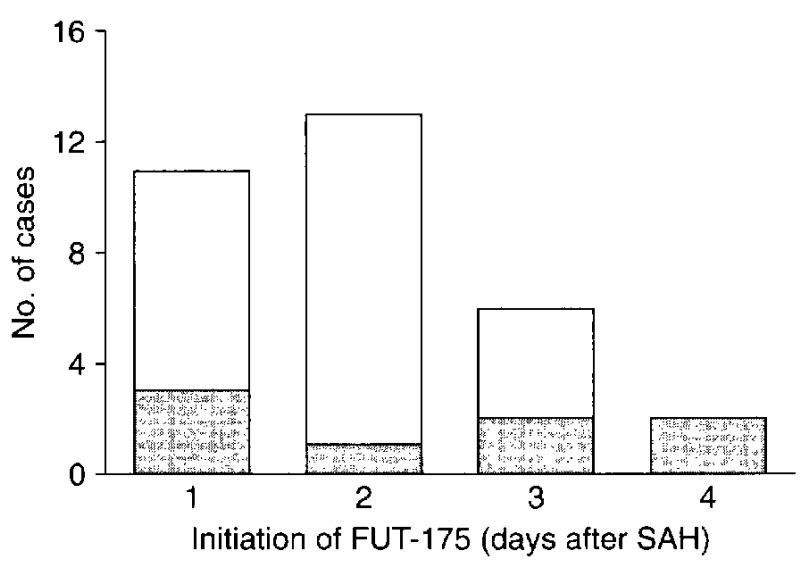

Fig. 2 Timing of FUT-175 therapy and incidence of symptomatic vasospasm. Patients received $\geq 80 \mathrm{mg} /$ day of FUT-175. open column: patients without symptomatic vasospasm, shaded column: patients with symptomatic vasospasm.

vasospasm. This difference was not significant. The significance of the timing of FUT therapy was also evaluated in patients treated with $80 \mathrm{mg} /$ day or more (Fig. 2). When FUT therapy was started within day 2 of $\mathrm{SAH}$, the incidence of symptomatic vasospasm was $16.7 \%$, but increased to $50 \%$ by late initiation of FUT therapy (start at day 3 or 4 after SAH). However, no statistical difference was demonstrated between these two groups. 


\section{Discussion}

This preliminary study of the preventive effects of FUT-175 examined patients with thick SAH on CT who were at greater risk to develop vasospasm. Our results suggest that administration of FUT-175 in addition to OKY-046 significantly decreases the incidence of symptomatic vasospasm in these high risk patients. Our results show that a dose of $80 \mathrm{mg} /$ day beginning on day 1 or 2 after SAH will achieve the optimum effect. Study of 304 patients with ruptured cerebral aneurysms in the anterior circulation treated surgically within 72 hours found that the incidence of symptomatic vasospasm was significantly different between patients with Hunt and Kosnik grades 2 and $3 .^{71}$ In our study in non-FUT patients with thick $\mathrm{SAH}$, symptomatic vasospasm also developed more frequently in Hunt and Hess grade 3 group than the grade 1 plus 2 group ( $p<0.001$ ). FUT-175 significantly reduced the incidence of vasospasm in these higher neurological grades (Table 2). Severe brain injury is primarily caused by $\mathrm{SAH}$ in patients with grade 5 , and our present study revealed the poor outcome for such patients in both the FUT and the non-FUT groups. Evaluation of the patients with Hunt and Hess grades 1-4 showed FUT-175 improved the outcome significantly $(\mathrm{p}<$ $0.05)$.

The therapeutic benefit of FUT-175 may be due both to preventing or ameliorating vasospasm and also to protecting neuronal elements against vasospasm-induced ischemia. Vasospasm usually develops between days 4 and 14 after SAH. The halflife of FUT-175 is short, ${ }^{12)}$ and FUT-175 treatment is initiated between days 1 and 4 after SAH and is used for only 4 days. Thus, the effect of FUT-175 probably does not continue till the late phase of vasospasm. Furthermore, the present study suggests that early initiation of FUT therapy is more effective to prevent symptomatic vasospasm. Therefore, FUT-175 is unlikely to reduce the incidence of DIND through neuronal protection or improvement of microcirculation in the ischemic area. Although we did not verify the effect of FUT-175 angiographically, we suggest that FUT-175 prevents symptomatic vasospasm through inhibition of the complement cascade during the acute stage of SAH.

These beneficial effect of FUT-175 appears to be additive to that of the thromboxane synthetase inhibitor, OKY-046. ${ }^{21)}$ We and our affiliated hospitals have been using OKY-046, which is a potent agent to prevent vasospasm between 1989 and 1994 as standard care to prevent or reduce DIND due to vasospasm. Clinical data from the 1905 patients treated were deposited in the Nagasaki SAH Data
Bank. Imbalance in the synthesis of $\mathrm{TXA}_{2}$ and prostacyclin results in vasoconstriction and platelet aggregation, in which intimal changes are involved. ${ }^{16]}$ Since intimal changes occur in the early stage of vasospasm, ${ }^{19)}$ this imbalance probably occurs after the initiation of vasospasm. Therefore, TXA $_{2}$ synthetase inhibitors may act to block the progression of vasospasm rather than prevent the initiation of vasospasm. This hypothesis is supported by the observation that enhancement of platelet function appears after day 5 in SAH patients with symptomatic vasospasm. ${ }^{17}$ Since we have no data on the outcome of patients with SAH not treated by OKY-046, we did not evaluate the effect of only FUT-175 treatment. However, this study suggests that the combined use of FUT-175, which may prevent the initiation of vasospasm, and OKY-046, which may act to inhibit progression of vasospasm, has an additive effect for the prevention of symptomatic vasospasm in high risk patients.

Hemorrhagic complications may be associated with OKY-046. ${ }^{21)}$ However, present study found no such complication in either the FUT or the non-FUT group, except one patient in the non-FUT group who died from acute subdural hematoma of unknown cause on day 12 of SAH. No other serious adverse effect of FUT-175 was experienced.

The combination of serine protease inhibitor FUT175 and $\mathrm{TXA}_{2}$ synthetase inhibitor OKY-046 can reduce the incidence of symptomatic vasospasm and improve the clinical outcome in patients with thick SAH on CT.

\section{References}

1) Asano T, Takakura K, Sano K, Kikuchi H, Nagai H, Saito I, Tamura A, Ochiai C, Sasaki T: Effects of a hydroxyl radical scavenger on delayed ischemic neurological deficits following aneurysmal subarachnoid hemorrhage: Results of a multicenter, placebo-controlled double-blind trial. J Neurosurg 84: 792-803, 1996

2) Buchheit F, Boyer P: Review of treatment of symptomatic cerebral vasospasm with nimodipine. Acta Neurochir Suppl (Wien) 45: 51-55, 1995

3) Fisher CM, Kistler JP, Davis JM: Relation of cerebral vasospasm to subarachnoid hemorrhage visualized by computerized tomographic scanning. Neurosurgery 6: 1-9, 1980

4) Grosset DG, McDonald I, Cockburn M, Straiton J, Bullock RR: Prediction of delayed neurological deficit after subarachnoid haemorrhage: A CT blood load and Doppler velocity approach. Neuroradiology 36: 418-421, 1994

5) Gurusinghe NT, Richardson AE: The value of computerized tomography in aneurysmal subarachnoid hemorrhage. The concept of the CT score. I Neu- 
rosurg 60: 763-770, 1984

6) Haley EC Jr, Kassell NF, Torner JC, the participants: A randomized controlled trial of high-dose intravenous nicardipine in aneurysmal subarachnoid hemorrhage: A report of the cooperative aneurysm study. J Neurosurg 78: 537-547, 1993

7) Hirai H, Ono J, Yamaura A: Clinical grading and outcome after early surgery in aneurysmal subarachnoid hemorrhage. Neurosurgery 39: 441-447, 1996

8) Hitomi Y, Fujii S: Inhibition of various immunological reactions in vivo by a new synthetic complement inhibitor. Int Arch Allergy AppI Immunol 69: 262267,1982

9) Hoshi T, Shimizu T, Kito K, Yamasaki N, Takahashi K, Takahashi M, Okada T, Kasuya H, Kitamura K: [Immunological study of late cerebral vasospasm in subarachnoid hemorrhage. Detection of immunoglobulins, C3, and fibrinogen in cerebral arterial walls by immunofluorescence method]. Neurol Med Chir (Tokyo) 24: 647-654, 1984 (Jpn, with Eng abstract)

10) Hughes JT, Schianchi PM: Cerebral artery spasm: A histological study at necropsy of the blood vessels in cases of subarachnoid hemorrhage. J Neurosurg 48: 515-525, 1978

11) Hunt WE, Hess RM: Surgical risk as related to time of intervention in the repair of intracranial aneurysms. J Neurosurg 28; 14-20, 1968

12) Ikari N, Sakai Y, Fujii S: New synthetic inhibitor to the alternative complement pathway. Immunology 49: 685-691, 1983

13) Jennett $B$, Bond $M$ : Assessment of outcome after severe brain damage. A practical scale. Lancet 1: 480484, 1975

14) Kassell NF, Haley EC Jr, Apperson-Hansen C, Alves WM, the participants: Randomized, double-blind, vehicle-controlled trial of tirilazad mesylate in patients with aneurysmal subarachnoid hemorrhage: A cooperative study in Europe, Australia, and New Zealand. J Neurosurg 84: 221-228, 1996

15) Kasuya H, Shimizu T: Activated complement components $\mathrm{C} 3 \mathrm{a}$ and $\mathrm{C} 4 \mathrm{a}$ in cerebrospinal fluid and plasma following subarachnoid hemorrhage. J Neurosurg 71: 741-746, 1989

16) Moncada S, Vane JR: Arachidonic acid metabolites and the interactions between platelets and blood vessel walls. $N$ Engl J Med 300: 1142-1147, 1979

17) Ohkuma H, Suzuki S, Kimura M, Sobata E: Role of platelet function in symptomatic cerebral vasospasm following aneurysmal subarachnoid hemorrhage. Stroke 22: 854-859, 1991

18) Pellettieri L, Nilsson B, Carlsson CA, Nilsson U: Serum immunocomplexes in patients with subarachnoid hemorrhage. Neurosurgery 19: 767771,1986

19) Seifert V, Stolke D, Reale E: Ultrastructural changes of the basilar artery following experimental subarachnoid haemorrhage: A morphological study on the pathogenesis of delayed cerebral vasospasm. Acta Neurochir (Wien) 100: 164-171, 1989
20) Shibuya M, Suzuki Y, Sugita K, Saito I, Sasaki T, Takakura K, Nagata I, Kikuchi H, Takemae T, Hidaka $\mathrm{H}$, Nakashima $\mathrm{M}$ : Effect of AT877 on cerebral vasospasm after aneurysmal subarachnoid hemorrhage: Results of a prospective placebo-controlled double-blind trial. J Neurosurg 76: 571-577, 1992

21) Suzuki S, Sano K, Handa H, Asano T, Tamura A, Yonekawa Y, Ono H, Tachibana N, Hanaoka K: Clinical study of OKY-046, a thromboxane synthetase inhibitor, in prevention of cerebral vasospasms and delayed cerebral ischaemic symptoms after subarachnoid hemorrhage due to aneurysmal rupture: A randomized double-blind study. Neurol Res 11: $79-88,1989$

22) Tanabe Y, Sakata K, Yamada H, Ito T, Takada M: Cerebral vasospasm and ultrastructural changes in cerebral arterial wall: An experimental study. J Neurosurg 49: 229-238, 1978

23) Yanamoto $\mathrm{H}$, Kikuchi H, Okamoto S: Effects of protease inhibitor and immunosuppressant on cerebral vasospasm after subarachnoid hemorrhage in rabbits. Surg Neurol 42: 382-387, 1994

24) Yanamoto $H$, Kikuchi H, Sato M, Shimizu Y, Yoneda S, Okamoto S: Therapeutic trial of cerebral vasospasm with the serine protease inhibitor, FUT175, administered in the acute stage after subarachnoid hemorrhage. Neurosurgery 30: 358363,1992

Address reprint requests to: M. Kaminogo, M.D., Department of Neurosurgery, Nagasaki University School of Medicine, 1-7-1 Sakamoto, Nagasaki 852-8501, Japan.

\section{Commentary}

This paper discusses the interesting part possibly played by inflammatory factors in the development of delayed vasospasm, presenting both histological and biochemical evidence in support. The use of FUT-175, an inhibitor of the complement system, has arisen from this. The results presented do go some way towards supporting this hypothesis, with a reduced incidence of symptomatic vasospasm in the group receiving FUT-175 as well as OKY-046, and an improved three-month outcome in this group for grade 1-4 patients.

However, I don't think one can be more definite than that at present, since this was not a randomized study, but the FUT-175 patients were compared with controls from presumably a number of centers in the district (concurrent or historical?). Also, even though the controls were also Fisher grade 3, the incidence of delayed spasm of more than $57 \%$ seems rather high. Confirmation of spasm by transcranial Doppler or an- 
giography in both groups would have been useful.

In the past, many different treatments for delayed vasospasm ${ }^{11}$ have appeared very promising when first used on a small number of patients without randomized controls receiving exactly the same background treatment, and then later controlled trials would show no effect. I think it very important that serious consideration be given to such a study at this stage in the development of FUT-175.

\section{Reference}

1) Wilkins RH: Attempted prevention or treatment of intracranial arterial spasm: a survey. Neurosurgery 6: 198-210, 1980

Nicholas W. C. DorSCH, F.R.C.S., F.R.A.C.S. Department of Neurosurgery Westmead Hospital Sydney, Australia

The authors studied the preventive effects of serine protease inhibitor, FUT-175, combined with OKY-046 for the prevention of symptomatic cerebral vasospasm. The study showed that the administration of FUT-175 with OKY-046 significantly decreased the incidence of symptomatic cerebral vasospasm and obtained a more favorable outcome compared with ad- ministration of OKY-046 alone.

There may be possible differences between two groups, FUT group and non-FUT group, in terms of surgical techniques, nutritional status, administration of other drugs like a $\mathrm{Ca}^{++}$antagonists and so on, because the background of each group is quite different. Comparison of clinical data between two groups is, therefore, not always proper. Other preventive measures used should also be taken into consideration in a comparative study of this sort.

The occurrence rate of symptomatic vasospasm in the non-FUT group (57\%) seems to correspond to that found in the natural course of subarachnoid hemorrhage, therefore, the superior clinical result in the FUT group may be caused simply by the effect of FUT. We would like to know whether there is any synergistic effect between two drugs. The preventive mechanisms of FUT-175, which is probably based on its anti-inflammatory effect, should also be investigated by clinical laboratory methods prior to and following treatment to analyze such factors as inflammatory products or reactions in the cerebrospinal fluid.

Shigeharu SuzuKI, M.D. Department of Neurosurgery Hirosaki University School of Medicine Aomori, Japan 